\title{
Prenatal echocardiography - the impact on neonatal management
}

\author{
IWONA JAŃCZEWSKA ${ }^{1}$ IWONA DOMŻALSKA-POPADIUK ${ }^{1}, Z B I G N I E W ~ S ́ W I A C T E K-B R Z E Z I N ́ S K I^{2}$ \\ 1 Department of Neonatology, Medical University of Gdańsk, Poland \\ 2 Department of Pediatric Cardiology and Congenital Heart Defects, Medical University of Gdańsk, Poland \\ Corresponding author \\ Iwona Jańczewska \\ Department of Neonatology \\ Medical University of Gdansk \\ Kliniczna 1 A, 80-402 Gdańsk, Poland \\ E-mail:ijan@gumed.edu.pl
}

\section{ABSTRACT}

Congenital heart disease (CHD) results in neonatal morbidity and mortality. Prenatal diagnosis allows preparing an appropriate perinatal and postnatal care. Babies born in low-risk level sites with unexpected CHD may have poorer outcomes. The purpose of this study was to compare results of foetal echocardiography to postnatal findings and assess the impact of antenatal suspicions of CHD on postnatal management. Medical records of mother-infant pairs with CHD admitted to the Neonatal Intensive Care Unit (NICU) of the Medical University of Gdansk from 01.01. to 31.12.2013 were reviewed. We analysed if the defect was detected pre- or postnatally, and if the diagnosis was made by the obstetrician from low-risk level sites (level I) or from a tertiary care centre (level II sonography). The overall incidence of CHD was $68(3,4 \%)$. Critical congenital heart defects (CCHD) were found in 24 neonates $(1,2 \%), 21$ were diagnosed prenatally, 3 were transferred from 1st level units.

Correlation between prenatal diagnosis made at our centre and postnatal findings was achieved in $47,7 \%$. Accuracy in all prenatal and postnatal findings for both I and II sonography levels was $35,2 \%$. There were major differences in the disproportion of the great vessels and postnatal confirmation of coarctation of the aorta (CoAo) $(7,1 \%)$. We obtained a high accuracy of prenatal and postnatal findings in detection of lesions such as Tetralogy of Fallot (ToF), transposition of the great arteries (TGA), DORV (double outlet right ventricle) and Critical Pulmonary Stenosis, which require an outflow tract view (92,9\% of cases). Conclusions: We confirmed increasing diagnostic rates when the diagnostics is performed at a tertiary care centre. These results are in agreement with literature stating that prenatal detection of CoAo is still challenging.

Despite the high rates of misdiagnosis, majority of infants benefited from prenatally diagnosed CCHD.

Key words: critical congenital heart disease, foetal, neonatal echocardiography, prenatal diagnosis.

\section{INTRODUCTION}

Congenital heart disease (CHD) results in significant neonatal morbidity and mortality. $(1,2)$

Prenatal diagnosis of CHD is increasingly common; it varies from 16 to $65 \%$ depending on the experience of the centre and that of the physician. (3) Previous studies have shown big disparities in detection rate between university centres and peripheral practices. Many countries attempted to introduce guidelines for foetal screening cardiac examination. Using a four chamber view is advocated for obstetric scan. But some lesions are not evident from this scanning plan. Without using the extended scan like three vessel view, some of severe $\mathrm{CHD}$, such as coarctation of the aorta (CoAo) remains undetected before birth. (4-6) Prenatal diagnosis allows to refer the parents to tertiary medical centres and prepare for planned delivery, as well as establish an appropriate perinatal and postnatal therapeutic plan. (7-9)

Duct dependent heart disease used to be considered as critical CHD (CCHD) in paediatric cardiology. In case of defects diagnosed after birth, after ductus arteriosus (DA) is closed, the infant that did not receive prostaglandin (PGE) was in a life-threatening situation. (10) When the defect is detected prenatally and the infant is given PGE as planned, the prognosis for good outcomes of the treatment becomes remarkably better. $(8,11,12)$ Prenatal assessment of severity of a cardiac disease seems to be the most important. On account of that, it can be planned whether the infant may require immediate intervention in the first hours after birth or can be stabilized with a PGE infusion and may not require a surgery in the first hours of life. $(8,11,12)$ Researches have recently approached to creating a classification of the management of foetuses in the delivery room, based on the prenatal diagnosis and the expected diagnosis at birth.

In Poland, Responek and Słodki defined four groups of CHD: the severest heart defects, severe urgent heart defects, severe planned heart defects and planned heart defects. (11) Berkley et al. proposed 5 care plans, depending on the expected severity of $\mathrm{CHD}$, starting from comfort care in case of lethal defects up to a delivery at a tertiary centre with planned delayed surgery at a tertiary cardiac centre or maternal transport with delivery at a tertiary cardiac centre.

In case when the prenatally expected severity of a defect and the suggested perinatal care plan are corresponding to the after-birth diagnosis, even if there are some differences in the anatomy of the defect, they can be classified as minor variations in prenatal and postnatal diagnosis. When the previously planned care program needs to be modified, it has to be classified as major variation. (12) Babies born in low-risk level sites with unexpected CHD may have significantly poorer outcomes due to lack of immediate access to appropriate speciality care. (13)

Prenatal suspicion of CHD, even if it turns out false positive, always instigates parental anxiety and requires detailed paediatric cardiac evaluation.

The purpose of this study was to compare 
results of foetal echocardiography (FE$\mathrm{CHO}$ ) to postnatal findings and to assess the impact of antenatal suspicions of CHD on postnatal management.

\section{METHODS}

Medical records of mother-infant pairs with CHD or other cardiac disorders admitted to the Neonatal Intensive Care Unit (NICU) of the Medical University of Gdansk from 01.01. to 31.12.2013 were reviewed.

We analysed if the defect was detected preor postnatally, and if the diagnosis was made by the obstetrician from low-risk level sites (level I) or from a tertiary care centre (level II sonography).

Foetuses referred to our tertiary perinatal care centre had been scanned by a perinatologist specialized in foetal medicine.

The types of CHD recognized prenatally were divided into groups based on the echocardiographic views required to identify the disease in the foetus. Group 1 were those malformations identified by a fourchamber view alone. Group 2 were those identified by the addition of an outflow tracts view. Group 3 were those defects which needed other views (table 1).

Disproportion of the great vessels was described separately. There were 28 prenatal suspicions of these anomalies.

Additionally, a separate group of others findings, like: cardiomegaly -2 , dextrocardia -1 , normal echo -2 , was isolated.

Postnatal confirmation was obtained on the basis of neonatal echocardiography (NECHO), performed by paediatric cardiologist before discharging the infants home or transferring to surgery centre. Late outcomes were obtained by reviewing medical records of paediatrics cardiac centre.

We compared the results of foetal and neonatal echocardiograms. Based on Berkley's suggestions, we classified differences as minor or major variations. A minor variation was the one that did not result in a treatment plan change, while a major variation did result in a treatment plan change. We looked at the accuracy of the prenatal and postnatal diagnosis in regards to guiding neonatal management after delivery. Arrhythmias without structural heart disease were excluded from the study.

\section{STATISTICAL ANALYSIS}

Accuracy of prenatal and postnatal diagnosis was evaluated by chi-square distri- bution and independence tests, using IBM SPSS 23.

\section{RESULTS}

The amount of cardiac disorders in neonates hospitalized in our facility (2008 infants) over the study period was $74(3,7 \%)$. Of them 68 were CHD $(3,4 \%$ of the whole group), in 6 newborns other cardiac disorders were detected (cardiac tumor in 1 infant, dextrocardia - 1 infant, false chordae tendineae - 1 and tricuspid insufficiency in 3 neonates). Severe and critical congenital heart defects were found in 24 neonates ( $1,2 \%$ of the series, $35,3 \%$ of all CHD), 21 were diagnosed prenatally at our centre, 3 were transferred from level I units. All infants with antenatal suspicion of cardiac lesions were admitted to the NICU and obtained cardiac assessment.

There were 88 foetuses in which an obstetric scan suggested cardiac lesions.

Of them 65 were verified at our centre. Remaining 23 were examined only at level I sonography.

Neonatal findings were similar with prenatal diagnosis in 31 cases; all of them were recognized at level II facility. There were 29 of $\mathrm{CHD}$ recognized. Minor variations were detected in 6 cases, all described as accurate. Additionally, $\mathrm{CHD}$ were excluded in 2 cases referred from level I facilities for foetal ECHO; NECHO revealed normal heart anatomy and they were assessed as accurate. Correlation between prenatal diagnosis made at level II sonography and postnatal findings was achieved in $47,7 \%$ of cases. Accuracy in all prenatal and postnatal findings for both I and II sonography levels was achieved in $35,2 \%$ of cases (table 2).

Accuracy of prenatal and postnatal diagnosis was $92,9 \%$ (13 cases from 14 foetuses) for heart defects requiring outflow view for detection, and 30,4\% (7 cases from 23 assessed foetuses) for those presented in four chamber view, as well as requiring other views for recognition - 33,3\% (6 cases among 18 foetuses). Regarding other diagnosis represented by 5 cases, there were 3 corrected ones (60\%) (tables 3.1-3.4) There were major differences in the disproportion of the great vessels and postnatal confirmation of CoAo; from 28 foetuses cardiac defects like Aortic Stenosis (AS) and CoAo were confirmed postnatally in only 2 cases $(7,1 \%)$ (table 3.5 ).

There were groups of $21 \mathrm{critical}$ and serious CHD detected prenatally, defined on the basis of a baby's after-birth condition, survival and timing of a surgical inter- vention: the severest (CHD impossible to treat), severe urgent cardiac disease (CHD needing invasive procedures within the first hours after birth), planned cardiac disease (CHD needing invasive procedures within the first months after birth) and planned heart defects (which do not require cardiac surgery within the first months after birth) (table 4). In one case a lethal cardiac defect was detected prenatally; the neonate's status deteriorated rapidly after birth, the baby received comfort care, yet died 2 hours after delivery. Nineteen neonates were admitted to urgent or planned surgery. There was hypoplastic left heart syndrome (HLHS) with intact atrial septum (IAS) diagnosed prenatally in 1 case with a satisfying atrial flow after birth, thus previously planned care program needed to be modified and the classification of a defect changed from severe urgent to severe planned (major variation). However, the infant required prostaglandin and a planned surgical intervention. The infant with Ebstein's anomaly and pulmonary atresia suspected prenatally as criss-cross heart despite of having major variation between foetal and neonatal ECHO benefited from prenatal suspicion of CCHD, because the prostaglandin intake has started immediately after birth. The neonate with corrected transposition of the great arteries (cTGA) plus ventricular inversion and atrio-ventricular block Wenckebach's type required antiarrhythmic treatment but did not require a surgery.

In cases of CCHD, prenatal diagnosis corresponded with postnatal findings in 19 of 21 foetuses diagnosed in our centre (accuracy 90,5\%) (table 4).

In the following prenatal examinations, 14 infants (13 of them examined at our centre) were diagnosed with cardiac disease with no instability expected including: mild pulmonary stenosis (PS) (1 baby); dextrocardia and Ventricular Septal Defect (VSD) (1 baby); dextrocardia (1 baby); VSD ( 3 babies); secundum atrial septal defect (ASD2) (1 baby); cardiac tumor without outflow obstruction (1 baby); tricuspid valve regurgitation (TVR) without other cardiac malformation (3 babies), dilated coronary sinus (1 baby) or bicuspid aortic valve (BAV) (1 baby).

In one neonate referred from level I facility for delivery at our centre clinically nonsignificant CHD (ASD2) occurred.

In 39 neonates cardiac defects were diagnosed after birth in neonatal echocardiography done for other reasons. Defects diagnosed postnatally are shown in table 5 . Three neonates without antenatal diagnosis of CCHD were referred to our centre 
Table 1. Division of congenital heart disease (CHD) into groups according to echocardiographic view required for prenatal diagnosis.

\begin{tabular}{llllll}
\hline Four-chamber view & \multicolumn{3}{l}{ Outflow tracts view } & \multicolumn{2}{l}{ Other view } \\
\hline Type of CHD & No of cases & Type of CHD & No of cases & Type of CHD & No of cases \\
\hline SV & 1 & $\begin{array}{l}\text { AS, VSD, disproportion of the } \\
\text { great vessels }\end{array}$ & 1 & $\begin{array}{l}\text { criss-cross heart, right sided aortic } 1 \\
\text { arch, PS, VSD }\end{array}$ & 1 \\
\hline AVSD & 2 & BAV & 2 & IAA type A, VSD & 1 \\
\hline HLHS & 3 & c-TGA & 1 & PLSVC & 1 \\
\hline VSD & 2 & d-TGA, ASD, VSD & 1 & PLSVC, CoAo, dextrocardia & 1 \\
\hline cardiac tumor & 14 & $\begin{array}{l}\text { Taussig-Bing syndrome, VSD } \\
\text { subpulmonic }\end{array}$ & 1 & Critical PS, underdevelopment & 1 \\
\hline & ToF & 5 & interrupted inferior vena cava & 1 \\
\hline & Critical PS & 1 & tricuspid valve regurgitation & 12 \\
\hline TOTAL & Mild PS & 1 & & & 18 \\
\hline
\end{tabular}

AS, Aortic stenosis; ASD2, secundum atrial septal defect; AVSD, atrioventricular septal defect; BAV, bicuspid aortic valve; CoAo, coarctation of the Aorta; c-TGA, corrected-TGA; DA, ductus arteriosus; DORV, double outlet right ventricle; d-TGA, dextro-transposition of the great arteries; HAA, hypoplastic aortic arch; HD, heart defect; HLHS, hypoplastic left heart syndrome; IAA, interrupted aortic arch; IAS, intact atrial septum; IVS, intact ventricle septum; l-TGA, levo-TGA; LVOTO, left venticular outflow tract obstruction; PAPVR, partial anomalous pulmonary venous return; PFO, patent foramen ovale; PA, pulmonary atresia; PS, pulmonary stenosis; PLSVC, persistent left superior vena cava; RV, Right Ventricle; ToF, tetralogy of Fallot; tAVC, transitional atrioventricular canal; VSD ventricular septal defect.

Table 2. Accuracy of foetal and neonatal echocardiography findings

\begin{tabular}{|c|c|c|c|}
\hline $\begin{array}{l}\text { Sonography level II } \\
\mathrm{n}=65\end{array}$ & & $\begin{array}{l}\text { Sonography level I } \\
\mathrm{n}=23\end{array}$ & $\begin{array}{l}\text { Sonography level } \\
\text { I and II } \\
\mathrm{n}=88\end{array}$ \\
\hline \multirow{2}{*}{$\begin{array}{l}\text { Accurate } \\
\mathrm{n}=31 \\
47,7 \%\end{array}$} & $\begin{array}{l}\text { Normal ECHO } \\
\mathrm{n}=2\end{array}$ & \multirow[t]{2}{*}{$\mathrm{n}=0$} & \multirow{2}{*}{$\begin{array}{l}\text { Accurate } \\
\mathrm{n}=31 \\
35,2 \%\end{array}$} \\
\hline & $\begin{array}{l}\mathrm{CHD} \\
\mathrm{n}=29\end{array}$ & & \\
\hline \multirow[t]{2}{*}{$\begin{array}{l}\text { Inaccurate } \\
\mathrm{n}=34 \\
52,3 \%\end{array}$} & $\begin{array}{l}\text { Major variation and Different non- } \\
\text { significant } \mathrm{CHD} \\
\mathrm{n}=6\end{array}$ & $\begin{array}{l}\text { Different } \\
\text { non-significant CHD } \\
\mathrm{n}=1\end{array}$ & \multirow[t]{2}{*}{$\begin{array}{l}\text { Inaccurate } \\
\mathrm{n}=57 \\
64,8 \%\end{array}$} \\
\hline & $\begin{array}{l}\text { No cardiac defects } \\
\mathrm{n}=28\end{array}$ & $\begin{array}{l}\text { No cardiac defects } \\
\mathrm{n}=22\end{array}$ & \\
\hline
\end{tabular}

CHD, congenital heart disease.

Tables 3.1-3.5. Accuracy of foetal echocardiography diagnosis (FECHO) and neonatal echocardiography diagnosis (NECHO) according to the echocardiographic view required for prenatal diagnosis.

Table 3.1. Four chamber view

\begin{tabular}{|c|c|c|c|c|c|c|}
\hline $\begin{array}{l}\text { No } \\
\text { of cases }\end{array}$ & FECHO diagnosis & Sonography level & NECHO diagnosis & $\begin{array}{l}\text { Accuracy } \\
\text { No of cases } \\
(\%)\end{array}$ & $\begin{array}{l}\text { Inaccuracy } \\
\text { No of cases } \\
(\%)\end{array}$ & Variation \\
\hline 1 & HLHS, intact IAS & II & HLHS, ASD2, DA & - & Inaccurate & Major \\
\hline 1 & $\begin{array}{l}\text { HLHS, Turner } \\
\text { Syndrome }\end{array}$ & II & HLHS, ASD, DA & Accurate & - & - \\
\hline 1 & $\begin{array}{l}\text { AS, single ventricle, } \\
\text { single atrium }\end{array}$ & & $\begin{array}{l}\text { AS, single ventricle, } \\
\text { single atrium }\end{array}$ & Accurate & - & - \\
\hline 1 & $\begin{array}{l}\text { AVSD, abnormal } \\
\text { karyotype }\end{array}$ & II & $\begin{array}{l}\text { VSD, Abnormal } \\
\text { karyotype }\end{array}$ & Accurate & - & Minor \\
\hline
\end{tabular}




\begin{tabular}{|c|c|c|c|c|c|c|}
\hline 2 & $\begin{array}{l}\text { AVSD, abnormal } \\
\text { karyotype }\end{array}$ & II & $\begin{array}{l}2 \times \text { AVSD, abnor- } \\
\text { mal karyotype }\end{array}$ & $2 \times$ Accurate & - & - \\
\hline 4 & VSD & II & $4 \mathrm{x}$ Normal echo & - & $4 \mathrm{x}$ Inaccurate & \\
\hline 1 & $\begin{array}{l}\text { VSD, abnormal } \\
\text { karyotype }\end{array}$ & II & $\begin{array}{l}\text { VSD, ASD2, Ar- } \\
\text { rhythmia, abnormal } \\
\text { karyotype }\end{array}$ & Accurate & - & - \\
\hline 2 & ASD2 & II & $2 \mathrm{x}$ Normal ECHO & - & $2 \mathrm{x}$ Inaccurate & - \\
\hline 1 & Cardiac tumor & II & Cardiac tumor & Accurate & - & - \\
\hline 9 & VSD & I & $9 \mathrm{x}$ Normal echo & - & $9 \mathrm{x}$ Inaccurate & - \\
\hline $\begin{array}{l}\text { SUM } \\
23\end{array}$ & & & & $\begin{array}{l}7 \\
(30,4 \%)\end{array}$ & $\begin{array}{l}16 \\
(69,6 \%)\end{array}$ & \\
\hline
\end{tabular}

AS, Aortic stenosis; ASD2, secundum atrial septal defect; AVSD, atrioventricular septal defect; BAV, bicuspid aortic valve; CoAo, coarctation of the Aorta; c-TGA, corrected-TGA; DA, ductus arteriosus; DORV, double outlet right ventricle; d-TGA, dextro-transposition of the great arteries; HAA, hypoplastic aortic arch; HD, heart defect; HLHS, hypoplastic left heart syndrome; IAA, interrupted aortic arch; IAS, intact atrial septum; IVS, intact ventricle septum; l-TGA, levo-TGA; LVOTO, left venticular outflow tract obstruction; PAPVR, partial anomalous pulmonary venous return; PFO, patent foramen ovale; PA, pulmonary atresia; PS, pulmonary stenosis; PLSVC, persistent left superior vena cava; RV, Right Ventricle; ToF, tetralogy of Fallot; tAVC, transitional atrioventricular canal; VSD ventricular septal defect.

Table 3.2. Outflow view

\begin{tabular}{|c|c|c|c|c|c|c|}
\hline No of cases & FECHO diagnosis & $\begin{array}{l}\text { Sonography } \\
\text { level }\end{array}$ & NECHO diagnosis & $\begin{array}{l}\text { Accuracy } \\
\text { No of cases } \\
(\%)\end{array}$ & $\begin{array}{l}\text { Inaccuracy } \\
\text { No of cases } \\
(\%)\end{array}$ & Variation \\
\hline 1 & $\begin{array}{l}\text { AS, VSD, Disproportion of I } \\
\text { the great vessels }\end{array}$ & & $\begin{array}{l}\text { AS, LVOTO, VSD, arrhythmia, } \\
\text { HF }\end{array}$ & Accurate & - & Minor \\
\hline 1 & ToF & II & DORV, VSD, tAVC, DA, PLSVC & Accurate & - & Minor \\
\hline 1 & ToF, right-sided aortic arch I & & $\begin{array}{l}\text { ToF, mild PS, right-sided aortic } \\
\text { arch }\end{array}$ & Accurate & - & - \\
\hline 3 & $\begin{array}{l}\text { ToF, hypoplastic pulmo- } \\
\text { nary artery }\end{array}$ & II & $\begin{array}{l}3 \times \mathrm{ToF} \text {, hypoplastic pulmonary } \\
\text { artery }\end{array}$ & $3 \times$ Accurate & - & - \\
\hline 1 & AVSD, PA & II & AVSD, PA, DORV & Accurate & - & Minor \\
\hline 1 & $\begin{array}{l}\text { Taussig-Bing Syndrome, } \\
\text { VSD subpulmonic, ar- } \\
\text { rhythmia }\end{array}$ & II & $\begin{array}{l}\text { ToF with critical PS } \\
\text { mesocardia }\end{array}$ & Accurate & - & Minor \\
\hline 1 & d-TGA, ASD, VSD & II & d-TGA, PFO, DA & Accurate & - & Minor \\
\hline 1 & c-TGA, arrhythmia & II & $\begin{array}{l}\text { c-TGA, ventricular inversion, } \\
\text { intact IVS, } \\
\text { L-TGA, AVB } 2 \text { degree }\end{array}$ & Accurate & - & - \\
\hline 1 & Critical PS & II & Critical PS & Accurate & - & - \\
\hline 1 & Mild PS & II & Mild PS & Accurate & - & - \\
\hline 1 & $\begin{array}{l}\text { Bicuspid Aortic Valve } \\
\text { (BAV) }\end{array}$ & II & BAV & Accurate & - & - \\
\hline 1 & $\begin{array}{l}\text { Bicuspid Aortic Valve } \\
\text { (BAV) }\end{array}$ & I & $1 \mathrm{x}$ Normal ECHO & - & Inaccurate & - \\
\hline $\begin{array}{l}\text { SUM } \\
14\end{array}$ & & & & $\begin{array}{l}13 \\
(92,9 \%)\end{array}$ & $\begin{array}{l}1 \\
(7,1 \%)\end{array}$ & \\
\hline
\end{tabular}

AS, Aortic stenosis; ASD2, secundum atrial septal defect; AVSD, atrioventricular septal defect; BAV, bicuspid aortic valve; CoAo, coarctation of the Aorta; c-TGA, corrected-TGA; DA, ductus arteriosus; DORV, double outlet right ventricle; d-TGA, dextro-transposition of the great arteries; HAA, hypoplastic aortic arch; HD, heart defect; HLHS, hypoplastic left heart syndrome; IAA, interrupted aortic arch; IAS, intact atrial septum; IVS, intact ventricle septum; l-TGA, levo-TGA; LVOTO, left venticular outflow tract obstruction; PAPVR, 
partial anomalous pulmonary venous return; PFO, patent foramen ovale; PA, pulmonary atresia; PS, pulmonary stenosis; PLSVC, persistent left superior vena cava; RV, Right Ventricle; ToF, tetralogy of Fallot; tAVC, transitional atrioventricular canal; VSD ventricular septal defect.

\section{Table 3.3. Other view}

\begin{tabular}{|c|c|c|c|c|c|c|}
\hline No of cases & FECHO diagnosis & Sonography level & NECHO diagnosis & $\begin{array}{l}\text { Accuracy } \\
\text { No of cases } \\
(\%)\end{array}$ & $\begin{array}{l}\text { Inaccuracy } \\
\text { No of cases } \\
(\%)\end{array}$ & Variation \\
\hline 1 & $\begin{array}{l}\text { Criss-cross heart, right-sided } \\
\text { aortic arch, PS, VSD, } \\
\text { HF, arrhythmia }\end{array}$ & & $\begin{array}{l}\text { c-TGA, PA, VSD, dextrocardia } \\
\text { with ventricular inversion, } \\
\text { Ebstein's anomaly, arrhythmia, } \\
\text { HF }\end{array}$ & & Inaccurate & Major \\
\hline 1 & IAA (type A), VSD & II & IAA (type B), VSD, PAPVR & Accurate & - & - \\
\hline 1 & $\begin{array}{l}\text { Critical PS, } \\
\text { underdevelopment of RV }\end{array}$ & II & $\begin{array}{l}\text { Critical PS, } \\
\text { Right Ventricle Hypoplasia, } \\
\text { PFO }\end{array}$ & Accurate & - & - \\
\hline 1 & PLSVC & II & Dilated coronary sinus-PLSVC & Accurate & - & - \\
\hline 1 & $\begin{array}{l}\text { PLSVC, CoAo, Dextrocardia, } \\
\text { SUA }\end{array}$ & & Dextrocardia, VSD, SUA & - & Inaccurate & Major \\
\hline 1 & $\begin{array}{l}\text { Interrupted inferior vena } \\
\text { cava }\end{array}$ & II & $1 \mathrm{x}$ Normal echo & - & Inaccurate & \\
\hline 1 & $\begin{array}{l}\text { Tricuspid Valve Regurgita- } \\
\text { tion } \\
\text { HF, ascites }\end{array}$ & II & $\begin{array}{l}\text { Tricuspid Valve Regurgitation } \\
\text { PFO, DA, no HF }\end{array}$ & Accurate & - & - \\
\hline 2 & $\begin{array}{l}\text { Tricuspid Valve Regurgita- } \\
\text { tion }\end{array}$ & II & $\begin{array}{l}2 \times \text { Tricuspid Valve Regurgita- } \\
\text { tion }\end{array}$ & - 2 x Accurate & & \\
\hline 1 & $\begin{array}{l}\text { Tricuspid Valve Regurgita- } \\
\text { tion }\end{array}$ & II & 1x normal ECHO & - & Inaccurate & \\
\hline 8 & $\begin{array}{l}\text { Tricuspid Valve Regurgita- } \\
\text { tion }\end{array}$ & I & $\begin{array}{l}8 \times \text { normal ECHO } \\
-\end{array}$ & - & $8 \mathrm{x}$ Inaccurate & \\
\hline $\begin{array}{l}\text { SUM } \\
18\end{array}$ & & & & $\begin{array}{l}6 \\
(33,3 \%)\end{array}$ & $12(66,7 \%)$ & \\
\hline
\end{tabular}

AS, Aortic stenosis; ASD2, secundum atrial septal defect; AVSD, atrioventricular septal defect; BAV, bicuspid aortic valve; CoAo, coarctation of the Aorta; c-TGA, corrected-TGA; DA, ductus arteriosus; DORV, double outlet right ventricle; d-TGA, dextro-transposition of the great arteries; HAA, hypoplastic aortic arch; HD, heart defect; HLHS, hypoplastic left heart syndrome; IAA, interrupted aortic arch; IAS, intact atrial septum; IVS, intact ventricle septum; l-TGA, levo-TGA; LVOTO, left venticular outflow tract obstruction; PAPVR, partial anomalous pulmonary venous return; PFO, patent foramen ovale; PA, pulmonary atresia; PS, pulmonary stenosis; PLSVC, persistent left superior vena cava; RV, Right Ventricle; ToF, tetralogy of Fallot; tAVC, transitional atrioventricular canal; VSD ventricular septal defect.

Table 3.4. Other diagnoses

\begin{tabular}{llllll}
\hline No of cases & FECHO diagnosis & Sonography level & NECHO diagnosis & $\begin{array}{l}\text { Accuracy } \\
\text { No of cases } \\
(\%)\end{array}$ & $\begin{array}{l}\text { Inaccuracy } \\
\text { No of cases } \\
(\%)\end{array}$ \\
\hline 1 & Dextrocardia & II & Dextrocardia & Accurate & - \\
\hline 2 & $\begin{array}{l}\text { Excluded CHD- } 2 \times \\
\text { normal FECHO }\end{array}$ & II & $2 \times$ Normal ECHO & $2 \times$ Accurate & - \\
\hline 2 & Cardiomegaly & I & $2 \times$ Normal echo & - & Inaccurate \\
\hline $\begin{array}{l}\text { SUM } \\
5\end{array}$ & & & 3 & $(60 \%)$ & 2 \\
\hline
\end{tabular}

CHD, congenital heart disease. 
Table 3.5. Disproportion of the great vessels

\begin{tabular}{|c|c|c|c|c|c|}
\hline No of cases & FECHO diagnosis & Sonography level & NECHO diagnosis & $\begin{array}{l}\text { Accuracy } \\
\text { No of cases } \\
(\%)\end{array}$ & $\begin{array}{l}\text { Inaccuracy } \\
\text { No of cases } \\
(\%)\end{array}$ \\
\hline 1 & $\begin{array}{l}\text { Disproportion of the } \\
\text { great vessels }\end{array}$ & II & $\begin{array}{l}\text { CoAo, HAA, DORV, } \\
\text { VSD, DA, BAV }\end{array}$ & Accurate & - \\
\hline 1 & $\begin{array}{l}\text { Disproportion of the } \\
\text { great vessels }\end{array}$ & II & CoAo, HAA, DA & Accurate & - \\
\hline 2 & $\begin{array}{l}\text { Disproportion of the } \\
\text { great vessels }\end{array}$ & II & $\begin{array}{l}\text { 1xVSD, PFO, DA } \\
\text { 1xASD2, DA, enlarge- } \\
\text { ment RV }\end{array}$ & - & $2 \mathrm{x}$ Inaccurate \\
\hline 21 & $\begin{array}{l}\text { Disproportion of the } \\
\text { great vessels }\end{array}$ & II & $21 \mathrm{x}$ normal ECHO & - & $21 \mathrm{x}$ Inaccurate \\
\hline 2 & $\begin{array}{l}\text { Disproportion of the } \\
\text { great vessels }\end{array}$ & I & $2 \mathrm{x}$ normal ECHO & - & $2 \times$ Inaccurate \\
\hline 1 & $\begin{array}{l}\text { Disproportion of the } \\
\text { great vessels }\end{array}$ & I & 1xASD2 & - & $1 \mathrm{x}$ Inaccurate \\
\hline $\begin{array}{l}\text { SUM } \\
28\end{array}$ & & & & $\begin{array}{l}2 \\
(7,1 \%)\end{array}$ & $\begin{array}{l}26 \\
(92,9 \%)\end{array}$ \\
\hline
\end{tabular}

AS, Aortic stenosis; ASD2, secundum atrial septal defect; AVSD, atrioventricular septal defect; BAV, bicuspid aortic valve; CoAo, coarctation of the Aorta; c-TGA, corrected-TGA; DA, ductus arteriosus; DORV, double outlet right ventricle; d-TGA, dextro-transposition of the great arteries; HAA, hypoplastic aortic arch; HD, heart defect; HLHS, hypoplastic left heart syndrome; IAA, interrupted aortic arch; IAS, intact atrial septum; IVS, intact ventricle septum; 1-TGA, levo-TGA; LVOTO, left venticular outflow tract obstruction; PAPVR, partial anomalous pulmonary venous return; PFO, patent foramen ovale; PA, pulmonary atresia; PS, pulmonary stenosis; PLSVC, persistent left superior vena cava; RV, Right Ventricle; ToF, tetralogy of Fallot; tAVC, transitional atrioventricular canal; VSD ventricular septal defect.

Table 4. Critical and Severe Cardiac Defects recognized following prenatal diagnosis (level II sonography)

\begin{tabular}{|c|c|c|c|}
\hline \multicolumn{2}{|c|}{$\begin{array}{l}\text { Severity of Type of CHD } \\
\text { CHD }\end{array}$} & \multirow{2}{*}{$\begin{array}{l}\text { Accuracy of Prenatal } \\
\text { diagnosis }\end{array}$} & \multirow{2}{*}{$\begin{array}{l}\text { Neonatal outcomes } \\
\text { Death }\end{array}$} \\
\hline $\begin{array}{l}\text { Severest } \\
(\mathrm{n}=1)\end{array}$ & AS, single ventricle, single atrium & & \\
\hline \multirow{2}{*}{$\begin{array}{l}\text { Severe } \\
\text { urgent HD } \\
(n=2)\end{array}$} & Critical PS & Accurate & Surgery \\
\hline & Critical PS, Right Ventricle Hypoplasia, PFO & Accurate & Surgery \\
\hline \multirow{12}{*}{$\begin{array}{l}\text { Severe } \\
\text { planned } \\
\text { HD }(n=14)\end{array}$} & CoAo, HAA, DA & Accurate & Surgery \\
\hline & CoAo, HAA, DORV, VSD, PDA, BAV & Accurate & Surgery \\
\hline & IAA (type B), VSD, PAPVR & Accurate & Surgery \\
\hline & AS, LVOTO, VSD, Arrhythmia, HF & Accurate & Surgery \\
\hline & HLHS, ASD2, DA & Accurate & Surgery \\
\hline & HLHS, ASD2, DA & $\begin{array}{l}\text { Major variation Inac- } \\
\text { curate }\end{array}$ & Surgery \\
\hline & DORV, VSD, tAVC, DA, PLSVC & Accurate & Surgery \\
\hline & AVSD, PA, DORV & Accurate & Surgery \\
\hline & ToF with critical PS, Mesocardia & Accurate & Surgery, death \\
\hline & $3 \mathrm{x}$ ToF, hypoplastic pulmonary artery & Accurate & $3 \mathrm{x}$ surgery \\
\hline & d-TGA, PFO, PDA & Accurate & \\
\hline & $\begin{array}{l}\text { c-TGA, PA, VSD, dextrocardia with ventricular inversion, } \\
\text { Ebstein's anomaly, arrhythmia, HF }\end{array}$ & Inaccurate & Blalock-Taussig shunt, palliative treatment \\
\hline
\end{tabular}




\begin{tabular}{llll}
\hline Planned & AVSD, abnormal karyotype & Accurate & Surgery \\
\cline { 2 - 4 } HD $(\mathrm{n}=3)$ & AVSD, abnormal karyotype & Accurate & Surgery, death \\
\cline { 2 - 4 } & ToF, mild PS, right-sided aortic arch & Accurate & Surgery \\
\hline Planned & c-TGA, ventricular inversion, intact IVS, L-TGA, AVB 2 & Accurate & Without surgery, antiarrhythmic treatment \\
HD $(n=1)$ & degree - no surgery & & \\
\hline Total $n=21$ & Accurate & \\
$(100 \%)$ & $(90,5 \%)$ & \\
& & $n=19$ & \\
\hline
\end{tabular}

AS, Aortic stenosis; ASD2, secundum atrial septal defect; AVSD, atrioventricular septal defect; BAV, bicuspid aortic valve; CoAo, coarctation of the Aorta; c-TGA, corrected-TGA; DA, ductus arteriosus; DORV, double outlet right ventricle; d-TGA, dextro-transposition of the great arteries; HAA, hypoplastic aortic arch; HD, heart defect; HLHS, hypoplastic left heart syndrome; IAA, interrupted aortic arch; IAS, intact atrial septum; IVS, intact ventricle septum; l-TGA, levo-TGA; LVOTO, left venticular outflow tract obstruction; PAPVR, partial anomalous pulmonary venous return; PFO, patent foramen ovale; PA, pulmonary atresia; PS, pulmonary stenosis; PLSVC, persistent left superior vena cava; RV, Right Ventricle; ToF, tetralogy of Fallot; tAVC, transitional atrioventricular canal; VSD ventricular septal defect.

Table 5. Cardiac defects identified postnatally.

\begin{tabular}{ll}
\hline Type of congenital heart disease & No of cases \\
\hline VSD & 22 \\
\hline ASD2 & 4 \\
\hline mild CoAo & 2 \\
\hline mild PS & 4 \\
\hline BAV & 3 \\
\hline PDA & 1 \\
\hline CoAo, HAA, VSD, PDA & 1 \\
\hline TGA, PDA, ASD & 1 \\
\hline ToF, hypoplastic PA, PFO & 1 \\
\hline
\end{tabular}

AS, Aortic stenosis; ASD2, secundum atrial septal defect; AVSD, atrioventricular septal defect; BAV, bicuspid aortic valve; CoAo, coarctation of the Aorta; c-TGA, corrected-TGA; DA, ductus arteriosus; DORV, double outlet right ventricle; d-TGA, dextro-transposition of the great arteries; HAA, hypoplastic aortic arch; HD, heart defect; HLHS, hypoplastic left heart syndrome; IAA, interrupted aortic arch; IAS, intact atrial septum; IVS, intact ventricle septum; l-TGA, levo-TGA; LVOTO, left venticular outflow tract obstruction; PAPVR, partial anomalous pulmonary venous return; PFO, patent foramen ovale; PA, pulmonary atresia; PS, pulmonary stenosis; PLSVC, persistent left superior vena cava; RV, Right Ventricle; ToF, tetralogy of Fallot; tAVC, transitional atrioventricular canal; VSD ventricular septal defect.

from other, non-tertiary units. They were diagnosed after delivery with CCHD: CoAo with hypoplastic aortic arch with ventricular septal defect and ductus arteriosus (CoAo/HAA/VSD/DA); transposition of the great arteries (dTGA/DA/ ASD2); and Tetralogy of Fallot (ToF) with hypoplastic pulmonary artery).

The neonate with concomitant CoAo and HAA was subjected to pulse oximetry test 3 hours after birth. According to medical history, the test yielded negative result. The baby remained at well-baby nursery for the first 2 days of life, until its status deteriorated (pallor, tachypnea, seizures, metabolic acidosis and kidney failure occurred). The infant needed to be intu- bated, required mechanical ventilation and a long-term stay at NICU. Despite the cardiac treatment, the outcomes were not satisfying and resulted in cerebral palsy, hydrocephalus and PVL (periventricular leukomalacia).

The neonate with dTGA/ASD/DA was born in a good general status; however, its status deteriorated shortly after birth. The baby was intubated and transferred to NICU with a suspicion of congenital pneumonia or heart defect. The patient was transferred immediately to a cardiosurgical centre; the result of surgical intervention was satisfactory.

The newborn with ToF was transferred to our tertiary centre due to respiratory fail- ure, evidence of heart murmur, dysmorphia and central nervous system anomalies (agenesis of the corpus callosum) and also needed to be intubated and required a long stay at NICU. It is not only a heart disease that influences his development, but also a defect of Central Nervous System (CNS).

\section{DISCUSSION}

Delayed diagnosis of CCHD leads to severe morbidity and mortality as many of these undiagnosed newborns become critically ill with cardiovascular collapse.

Prenatal diagnosis of CHD by foetal echocardiography is now a firmly established 
component of foetal medicine offered in many tertiary medical centres. Despite the fact, the proportion of CHD detected prenatally remains low internationally. (14) In many facilities in the United States less than $50 \%$ of neonates with CHD are diagnosed antenatally. $(7,15,16)$

In Europe, major differences in detection rate in various countries were observed. The highest detection rates were observed in France (48\%), Spain (45\%) and Germany $(40 \%)$, while in Eastern Europe (Croatia, Lithuaniaand Ukraine) the detection rate equalled 8\%. (14) Mean European percentage of detected CHD was calculated to be $25 \%$. (14) Marek J et al. assessed prevalence of $\mathrm{CHD}$ based on nationwide prenatal ultrasound screening programme in Czech Republic at 28,5\% in 2003. (17)

Regarding the diagnoses made at our centre, overall prenatal detection rate reached $47,7 \%$. These results placed us in the middle of mean European detection rate. Some previous studies showed similar antenatal detection rate. $(3,16)$ Mark K Friedberg stated the accuracy of $36 \%$ in an academic centre. (16) Acherman et al. showed prenatal detection rate of $36 \%$ for CCHD in Southern Nevada, but after developing community-wide foetal cardiology program, detection rate increased to $71 \%$. $(5,18)$ Many other scientific descriptions are also stating the increasing percentage of prenatal detections, especially at university facilities. $(12,19-22)$ So these numbers need to be improved. We analysed all prenatal suspicions made at any medical setting and at any gestational age, so the combined accuracy of pre- and postnatal findings for both level I and level II sonography was $35,2 \%$. Previous studies have also noted a higher prenatal detection rate made at tertiary-care centres then in lowlevel facilities. (19-21) Mark K Friedberg indicated $80 \%$ of compatibility of diagnosis in academic centres versus $23 \%$ in other practices. (16) It had been described that examination of foetus with four chamber view, used as an obstetrical screening of CHD, allowed detecting about $40 \%$ of CHD. $(6,23)$ In our study, defects requiring four chamber view were identified in only $30,4 \%$ of cases. We found low detection rate for septal defects, none of 9 VSD suspected in level I sonography centre and of 1 suspected in level II centre was proved in neonatal echocardiograpy. We observed better correlation in recognition of AVSD. Our observations are similar to the results obtained by Garne et al. evaluating data from 20 European registries. (14)

All cases of HLHS found in our study were detected prenatally. Of 2 cases of suspected HLHS, 2 were proved after delivery. Other researchers also stated good detection rate of HLHS (four chamber abnormality). $(16,24)$ There was a much closer correlation observed in our centre in detection of lesions such as Tetralogy of Fallot, Transposition of the Great Arteries, Double Outlet Right Ventricle and Critical Pulmonary Stenosis, which require an outflow tract view. Contrary to the literature that demonstrates low detection rate, we obtained a higher accuracy of prenatal and postnatal findings in these defects $(92,9 \%$ of cases). Friedberg et al. showed 19\% of TGA diagnosed prenatally while Allan noted detection rate for TGA 3\% (outflow tract view abnormality). (16,25)

The results of our study can be explained by the fact that all of the described cases were examined in level II sonography unit. Data from the literature also indicate the higher detection rate for defects requiring outflow view in academic centres. (16) Although some lesions can be accurately diagnosed by foetal echocardiography, prenatal detection of CoAo is still challenging. Many authors defined CoAo as the most common misdiagnosis. $(4,16,26)$ Our observations are consistent with the results of other studies. We showed major differences in the disproportion of the great vessels and postnatal confirmation of CoAo (7.1\%).

The importance of antenatal detection of CCHD cannot be understated. Early and accurate prenatal diagnosis allows optimizing medical care for mother and her child after birth. A multidisciplinary, specialized care team including an obstetrician, a neonatologist and a paediatric cardiologist may be useful in improving effects and late outcomes of treatment. $(8,12,22,24,27,28)$ Arrangement of an appropriate management and delivery plan based on foetal echo is even more important than the accuracy of pre- and postnatal findings. (29) In our work we detected 3 major variations, including one prenatal HLHS with IAS, which classified the finding as the severest cardiac defect. Satisfying interatrial communication was discovered after birth, classification of the defect was modified to severe planned. However, the baby benefited from prenatal diagnosis because s/he was delivered at a tertiary NICU where specialized care was taken immediately after birth. This enabled us to start an immediate implementation of an appropriate treatment, namely maintenance of patent ductus arteriosus by administration of prostaglandins and planned refer- ral for surgery prior to development of lifethreatening consequences of the defect. Many papers showed that prenatal diagnosis of cardiac disease as HLHS, CoAo or TGA, reduced early neurologic morbidity and improved long-term neurologic outcomes. (13,30-32) Of 24 CCHD detected in our group, 21 were diagnosed on the basis of foetal echocardiography. Nineteen neonates diagnosed and delivered at our centre were referred for planned surgery based on foetal and neonatal ECHO findings. Such attitude is associated with better long-term outcomes, and, most of all, with greater likelihood of normal neurological development in these infants. Infants with congenital heart disease without prenatal diagnosis, born in low-level facilities, are at risk of not receiving a needed care plan and, in the end, of worse treatment outcomes. $(12,13,30)$ Delayed diagnosis in patients who survive is the risk of hypoxic/ ischaemic brain injury. Periventricular leukomalacia has been reported on MRI imaging of the brain in up to $39 \%$ of neonates with critical CHD. (33) Prenatal diagnosis is associated with lower rate of acidosis, less frequent neurological complications and planned referral for surgery prior to development of life-threatening consequences of the defect, while a delay in diagnosis may lead to hypoxia, shock, multiorgan failure and worse outcomes of both early and late surgical actions. $(13,30,33)$ These can be confirmed by our observations. Infant with a CoAo and HAA diagnosed after birth was transferred to our unit in shock, with acidosis, needed to be intubated and required mechanical ventilation. Late outcomes of the treatment are not satisfying as it can result in cerebral palsy and periventricular leukomalacia.

Notwithstanding the higher prenatal detection rate of $\mathrm{CHD}$ done at a tertiary setting, foetal cardiac assessment is still performed at level I sonography, as pregnant women are not referred for foetal echo from the primary services. $(3,20)$ False positive diagnosis of foetal cardiac abnormality results in parents' anxiety, in contrast to misdiagnosis of CCHD in foetus, thereby delaying the diagnosis and, consequently, adequate specialized prenatal follow-up and delivery management at a specialized centre. It should be emphasized that infants born at our centre had prenatal diagnosis of CCHD, in contrast to those born at other sites, which affected postnatal management and late outcomes. Neonates with CCHD born at non-tertiary centres weretransferred to our unit, which implies that availability of prenatal diagno- 
sis beyond university clinics is still insufficient. Newborns with late diagnosis of a CCHD were referred to cardiosurgical treatment in poor general and neurological status, which is another argument for identification of an accurate method for early detection of critical heart defect.

Conclusions
The prenatal diagnosis has significant impact on neonatal management, allowing appropriate medical care and planning surgery immediately after birth.

We confirmed increasing diagnostic rates when the diagnostics is performed at a tertiary care centre. The results of our study are in agreement with literature which states that prenatal detection of CoAo is still challenging.

Despite the high rates of misdiagnosis, majority of infants benefited from prenatally diagnosed CCHD. Improved accuracy in foetal diagnosis can be achieved through better organisation of perinatal care.

\section{REFERENCES}

1. Meberg A, Lindberg H, Thaulow, E. Congenital heart defects: The patients who die. Acta Paediatr 2007;94(8):1060-65. http://doi. org/10.1111/j.1651-2227.2005.tb02046.x

2. Khoshnood B, Lelong N, Houyel L, Thieulin AC, Jouannic JM, Magnier S, et al. Prevalence, timing of diagnosis and mortality of newborns with congenital heart defects: a population-based study. Heart 2012;98(22):1667-73.

3. Pinto NM, Keenan HT, Minich LL, Puchalski MD, Heywood M, Botto LD. Barriers to prenatal detection of congenital heart disease: a population-based study. Ultrasound Obstet Gynecol 2012;40(4):418-25. http://doi.org/10.1002/uog.10116

4. Buyens A, Gyselaers W, Coumans A, Al Nasiry, S, Willekes C, Boshoff, D, et al. Difficult prenatal diagnosis: fetal coarctation. Facts Views Vis Obgyn. 2012;4(4):230-6. Retrieved from http://www.ncbi.nlm.nih.gov/pubmed/24753914

5. Evans W, Castillo W, Rollins R, Luna C, Kip K, Ludwick J, et al Moving towards universal prenatal detection of critical congenital heart disease in southern Nevada: a community-wide program. Pediatr Cardiol 2015;36(2):281-8. doi:10.1007/s00246-014-0996-1.

6. Hill GD, Block JR, Tanem JB, Frommelt MA. Disparities in the prenatal detection of critical congenital heart disease. Prenat Diagn 2015;35(9):859-63. doi:10.1002/pd.4622.

7. Colby C. E, Carey WA, Blumenfeld YJ, Hintz SR. Infants with prenatally diagnosed anomalies: special approaches to preparation and resuscitation. Clin Perinatol 2012;39(4):871-87. http://doi.org/10.1016/j.clp.2012.09.012

8. Donofrio MT, Levy RJ, Schuette JJ, Skurow-Todd K, Sten M-B, Stallings C, et al. Specialized delivery room planning for fetuses with critical congenital heart disease. Am J Cardiol 2013;111(5):737-47. http://doi.org/10.1016/j.amjcard.2012.11.029

9. Allan LD, Huggon IC. Counselling following a diagnosis of congenital heart disease. Prenat Diagn 2004;24(13):1136-42. http:// doi.org/10.1002/pd.1071

10. Meberg A.Critical heart defects-the diagnostic challenge. Acta Paediatr.2008;97(11):1480-83.doi:10.1111/j.1651-2227.2008.00975.x.

11. Słodki M, Respondek-Liberska M, Pruetz JD, Donofrio MT. 2016; Fetal cardiology: changing the definition of critical heart disease in the newborn. J Perinatol 36(8): 575-80. http://doi.org/10.1038/jp.2016.20

12. Berkley EMF, Goens MB, Karr S, Rappaport V. Utility of fetal echocardiography in postnatal management of infants with prenatally diagnosed congenital heart disease. Prenat Diagn 2009;29(7):654-8. http://doi.org/10.1002/pd.2260

13. Tworetzky W, McElhinney DB, Reddy VM, Brook MM, Hanley FL, Silverman NH. Improved surgical outcome after fetal diagnosis of hypoplastic left heart syndrome. Circulation 2001;103(9):1269-73. http://www.ncbi.nlm.nih.gov/pubmed/11238272.

14. Garne E, Stoll C, Clementi M, Euroscan Group. Evaluation of prenatal diagnosis of congenital heart diseases by ultrasound: experience from 20 European registries. Ultrasound Obstet Gynecol 2001;17(5):386-91. http://doi.org/10.1046/j.1469-0705.2001.00385.x

15. Oster ME, Kim CH, Kusano AS, Cragan JD, Dressler P, Hales AR, et al. A population-based study of the association of prenatal diagnosis with survival rate for infants with congenital heart defects. Am J Cardiol 2014;113(6):1036-40. http://doi.org/10.1016/j. amjcard.2013.11.066

16. Friedberg MK, Silverman NH, Moon-Grady AJ, Tong E, Nourse J, Sorenson B, et al. Prenatal detection of congenital heart disease. J Pediatr 2009;155(1):26-31.e1. http://doi.org/10.1016/j.jpeds.2009.01.050

17. Marek J, Tomek V, Skovránek J, Povysilová V, Samánek M. Prenatal ultrasound screening of congenital heart disease in an unselected national population: a 21-year experience. Heart 2011;97(2):124-30. doi:10.1136/hrt.2010.206623.

18. Acherman RJ, Evans WN, Luna CF, Rollins R, Kip K, Collazos J, et al.Prenatal detection of congenital heart disease in southern Nevada: the need for universal fetal cardiac evaluation. J Ultrasound Med 2007;26(12):1715-9-1. http://www.ncbi.nlm.nih.gov/ pubmed/18029923.

19. Dolk H, Loane M, Garne E. Congenital heart defects in Europe: prevalence and perinatal mortality, 2000 to 2005 . Circulation 2011;123(8):841-9. doi:10.1161/CIRCULATIONAHA.110.958405.

20. Trivedi N, Levy D, Tarsa M, Anton T, Hartney C, Wolfson T, et al. Congenital cardiac anomalies: prenatal readings versus neonatal outcomes. J Ultrasound Med 2012;31(3):389-99. Retrieved from http://www.ncbi.nlm.nih.gov/pubmed/22368129

21. Meyer-Wittkopf M, Cooper S, Sholler G. Correlation between fetal cardiac diagnosis by obstetric and pediatric cardiologist sonographers and comparison with postnatal findings. Ultrasound Obstet Gynecol 2001;17(5):392-7. http://doi.org/10.1046 /j.1469-0705.2001.00381.

22. Friedman AH, Kleinman CS, Copel JA. Diagnosis of cardiac defects: where we've been, where we are and where we're going. Prenat Diagn 2002;22(4):280-4. http://doi.org/10.1002/pd.305

23. Gonçalves LF, Bronsteen R, Lee W. Fetal heart: a 4-chamber view is not enough. Clin Obstet Gynecol 2012;55(1):266-280. doi:10.1097/GRF.0b013e3182446df0.

24. Simpson JM. Impact of fetal echocardiography. Ann Pediatr Cardiol 2009;2(1):41-50. doi:10.4103/0974-2069.52806.

25. Allan L, Dangel J, Fesslova V, Marek J, Mellander M, Oberhansli I, et al. Recommendations for the practice of fetal cardiology in Europe. Cardiol Young 2004;14(1):109-14. http://www.ncbi.nlm.nih.gov/pubmed/15237685. 
26. Slodki M, Rychik J, Moszura T, Janiak K, Respondek-Liberska M. Measurement of the great vessels in the mediastinum could help distinguish true from false-positive coarctation of the aorta in the third trimester. J Ultrasound Med 2009;28(10):1313-17. http:// www.ncbi.nlm.nih.gov/pubmed/19778876.

27. Chung ML, Lee BS, Kim EA-R, Kim K-S, Pi S-Y, Oh YM, et al. Impact of fetal echocardiography on trends in disease patterns and outcomes of congenital heart disease in a neonatal intensive care unit. Neonatology 2010;98(1):41-6. http://doi.org/10.1159/000264673

28. Clur SA, Van Brussel PM, Ottenkamp J, Bilardo CM. Prenatal diagnosis of cardiac defects: accuracy and benefit. Prenat Diagn 2012;32(5):450-5. doi:10.1002/pd.3837.

29. Holland BJ, Myers JA, Woods CR. Prenatal diagnosis of critical congenital heart disease reduces risk of death from cardiovascular compromise prior to planned neonatal cardiac surgery: a meta-analysis. Ultrasound Obstet Gynecol 2015;45(6):631-8. doi:10.1002/ uog.14882.

30. Mahle WT, Clancy RR, McGaurn SP, Goin JE, Clark BJ. Impact of prenatal diagnosis on survival and early neurologic morbidity in neonates with the hypoplastic left heart syndrome. Pediatrics 2001;107(6):1277-82. http://www.ncbi.nlm.nih.gov/pubmed/11389243.

31. Franklin O, Burch M, Manning N, Sleeman K, Gould S, Archer N. Prenatal diagnosis of coarctation of the aorta improves survival and reduces morbidity. Heart 2002;87(1):67-9. http://www.ncbi.nlm.nih.gov/pubmed/11751670.

32. Escobar-Diaz MC, Freud LR, Bueno A, Brown D, Friedman K, Schidlow D, et al. Prenatal diagnosis of transposition of the great arteries over a 20-year period: improved but imperfect. Ultrasound Obstet Gynecol 2015;45(6):678-82. doi:10.1002/uog.14751.

33. McGovern E, Sands AJ. Perinatal management of major congenital heart disease. Ulster Med J 2014;83(3):135-9. http://www.pubmedcentral.nih.gov/articlerender.fcgi?artid=4255832\&tool=pmcentrez\&rendertype=abstract. Accessed January 4, 2016. 